\title{
Encouraging results for belatacept immunosuppression in renal transplantation
}

a mmunosuppression with belatacept, a fusion human protein that acts as

a blocker of T-cell costimulation, is associated with similar patient-survival and graft-survival rates as treatment with ciclosporin, according to the preliminary results of two phase III studies of renal transplant recipients. These studies also indicate that belatacept treatment is associated with reduced parameters of cardiovascular risk.

Cardiovascular disease is the leading cause of 1-year mortality in renal transplant recipients. Calcineurin inhibitors, such as ciclosporin or tacrolimus, exacerbate hypertension, diabetes, and dyslipidemia-which are risk factors for cardiovascular morbidity and mortality. Immunosuppressants that have milder adverse effects than calcineurin inhibitors and are as effective are therefore very desirable.

According to Flavio Vincenti, University of California, San Francisco, previous studies demonstrated robust immunosuppressive effects associated with the blockade of T-cell costimulation in rodents and non-human primates. Moreover, in a phase II study of adult renal transplant recipients, belatacept was associated with improved renal function and similar acute rejection rates as administration of ciclosporin.

The preliminary results of two large, randomized, multicenter phase III trials that compared two different regimens of belatacept therapy with ciclosporin therapy in adult recipients of renal transplants from living or standardcriteria deceased donors (BENEFIT) or from extended-criteria donors (BENEFIT-EXT) have now been reported. Both BENEFIT $(n=686)$ and BENEFITEXT $(n=578)$ randomly assigned renal transplant recipients to receive a more-intensive belatacept regimen, a less-intensive belatacept regimen, or a ciclosporin regimen. BENEFIT and BENEFIT-EXT will collect clinical data for

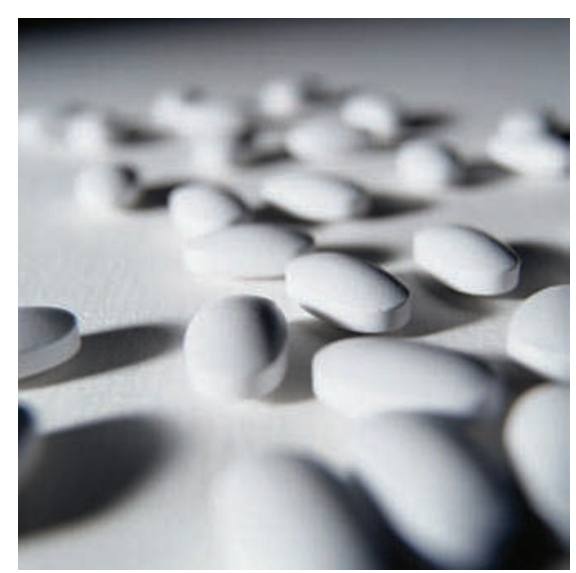

3 years after transplantation, but results after 1 year have now been published.

BENEFIT and BENEFIT-EXT participants treated with the two belatacept regimens had similar patientsurvival and graft-survival rates as their counterparts treated with ciclosporin. Patients in BENEFIT treated with the more-intensive belatacept regimen had a higher incidence of acute rejection than those treated with ciclosporin $(22 \%$ versus $7 \%$ ). The discrepancy in rates of acute rejection did not, however, affect the overall rates of patient survival (moreintensive $95 \%$; less-intensive $97 \%$; and ciclosporin 93\%) or graft loss (moreintensive $2 \%$; less-intensive $2 \%$; and ciclosporin, $4 \%$ ).

Fewer patients treated with the moreintensive and the less-intensive belatacept regimens in BENEFIT and with the moreintensive belatacept regimen in BENEFITEXT had renal function impairment than patients treated with ciclosporin. Moreover, the average measured glomerular filtration rate was significantly higher in BENEFIT participants treated with belatacept than in BENEFIT participants treated with ciclosporin (by $\sim 14 \mathrm{ml} / \mathrm{min} / 1.73 \mathrm{~m}^{2}$ ), and a similar trend was observed in BENEFIT-EXT.

In both BENEFIT and BENEFIT-

EXT, belatacept administration was associated with lower average diastolic and systolic blood pressure than ciclosporin administration. In addition, in patients receiving belatacept, average serum levels of triglycerides decreased from baseline, whereas they increased in patients treated with ciclosporin. A trend toward a lower incidence of new-onset diabetes after transplantation was also observed in patients receiving belatacept than in those receiving ciclosporin. Finally, a trend for an increased incidence of post-transplant lymphoproliferative disorder was noticed in patients treated with belatacept, particularly in those with known risk factors for the disease. Vincenti, a coauthor of both BENEFIT and BENEFITEXT, therefore suggests that belatacept use should be avoided in patients with negative serology for Epstein-Barr virus, who are at increased risk of post-transplant lymphoproliferative disorder.

According to the BENEFIT researchers, although the 1-year patient-survival and graft-survival rates in the belatacepttreated group were similar to those of the ciclosporin-treated group, several studies demonstrated a positive association between renal function 1 year after transplantation and long-term outcomes. The BENEFIT-EXT researchers say that the decrease in blood pressure and serum triglyceride levels in patients treated with belatacept compared with their ciclosporin-treated counterparts indicates that patients who received belatacept may be at decreased risk of cardiovascular mortality.

The BENEFIT researchers aim to assess these outcomes over the next 2 years.

\section{Baldo Lucchese}

\footnotetext{
Original articles Vincenti, F. et al. A phase III study of belatacept-based immunosuppression regimens versus cyclosporine in renal transplant recipients (BENEFIT study). Am. J. Transplant. 10, 535-546 (2010) | Durrbach, A. et al. A phase III study of belatacept versus cyclosporine in kidney transplants from extended criteria donors (BENEFITEXT study). Am. J. Transplant. 10, 547-557 (2010)
} 